\title{
VALENCY OF Yb IN PbS AND PbTe DETERMINED BY XPS
}

\author{
Z. GolaCKI \\ Institute of Physics, Polish Academy of Sciences \\ Al. Lotników 32/46, 02-668 Warsaw, Poland \\ AND M. HEINONEN \\ Department of Applied Physics, University of Turku, ElectroCity \\ Tykistokatu 2D, 20520 Turku, Finland

\begin{abstract}
$\mathrm{X}$-ray photoelectron spectroscopy experiments on $\mathrm{PbTe} \mathrm{Yb}, \mathrm{PbS}: \mathrm{Yb}$, $\mathrm{PbTe}$, and $\mathrm{PbS}$ crystals have been performed using the monochromatized Al $K_{\alpha}$ radiation. The valence band spectrum of $\mathrm{PbTe}: \mathrm{Yb}$ exhibits one set of peaks associated with divalent $\mathrm{Yb}$ initial states, but two sets associated with divalent and trivalent $\mathrm{Yb}$ are observed for $\mathrm{PbS}: \mathrm{Yb}$. The valency of $\mathrm{Yb}$ in $\mathrm{PbTe}: \mathrm{Yb}$ is 2 (within an accuracy of the experiment) but in $\mathrm{PbS}: \mathrm{Yb}$ the mixed valency of $\mathrm{Yb}$ is seen. These conclusions are confirmed by an analysis of $\mathrm{Yb} 4 d$ spectra in $\mathrm{PbTe}: \mathrm{Yb}$ and $\mathrm{PbS}: \mathrm{Yb}$ crystals.
\end{abstract}

PACS numbers: $71.20 . \mathrm{Nr}, 79.60 .-\mathrm{i}$

Ytterbium compounds show many interesting physical phenomena, e.g., Kondo effect, mixed valence, and existence of heavy fermions [1-3]. Yb monochalcogenides crystallize in the rock salt structure similarly as lead chalcogenides and form with them the mixed solid solutions. Depending on the host matrix the environment of $\mathrm{Yb}$ ions changes ( $\mathrm{S}, \mathrm{Se}$ or $\mathrm{Te}$ ) and the lattice constant changes considerably. The lattice constant usually influences (chemical pressure) the valency of the ions with the tendency to induce a mixed valence (Ce, Sm, Eu, Tm and $\mathrm{Yb}$ ). In the presented paper the effort was done to determine by X-ray photoelectron spectroscopy (XPS) the valency of $\mathrm{Yb}$ ions in $\mathrm{PbTe}$ and $\mathrm{PbS}$, which is not obvious and need the experimental proof.

$\mathrm{PbYbTe}, \mathrm{PbYbS}, \mathrm{PbTe}$, and $\mathrm{PbS}$ crystals were grown by the Bridgman method. $\mathrm{Yb}$ content was determined by the electron microprobe analysis to be equal 2 at.\% for $\mathrm{PbS}: \mathrm{Yb}$ and 3 at.\% for PbTe:Yb. PbS:Yb samples were of $n$-type, $\mathrm{PbTe}: \mathrm{Yb}$ samples were of $p$-type. No inclusions were found in the samples. The XPS spectra were recorded using a Perkin-Elmer small spot ESCA spectrometer equipped with an X-ray $\mathrm{Al}$ tube, a Rowland circle monochromator, and a hemispherical electron energy analyser. The spectral resolution was $0.7 \mathrm{eV}$. Samples were introduced into the preparation chamber with a vacuum better than 
$10^{-9}$ torr, cleaved, and then shifted to the measuring chamber with a vacuum better than $5 \times 10^{-10}$ torr. $\mathrm{Pb} 4 f$ and $5 d$, Te $3 d$ and $4 d$, and S $2 p$ spectra were measured for all the crystals (for the control of the experiment), but the attention was paid mainly to the valence band (VB) spectra and the $4 d$ spectra of $\mathrm{Yb}$ ions. The experimental conditions - photon energy $1486.6 \mathrm{eV}$ favour the bulk contribution of the photoelectron process.

The VB spectra of $\mathrm{PbTe}$ and $\mathrm{PbS}$ registered in this work are similar to those reported earlier in the literature [4]. The core levels spectra of $\mathrm{Pb}, \mathrm{Te}$, and $\mathrm{S}$ did not show any pronounced changes with doping by $\mathrm{Yb}$.

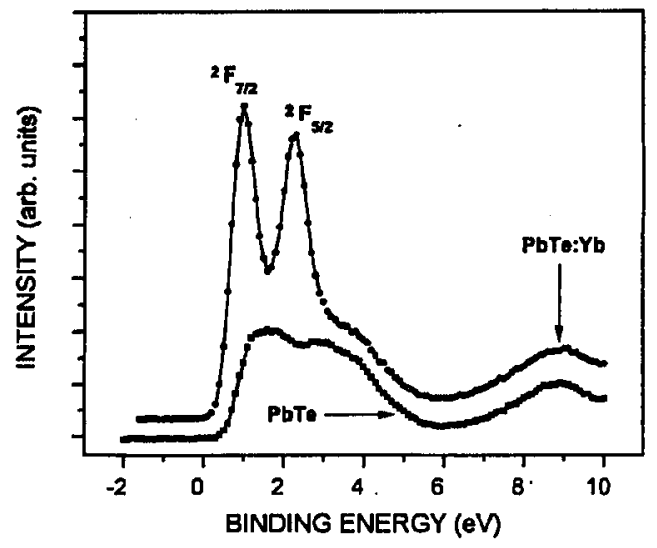

Fig. 1. XPS valence band spectra for $\mathrm{PbTe}$ and $\mathrm{PbTe} \mathrm{Yb}$.

Figure 1 shows the normalized VB photoemission spectra of $\mathrm{PbTe}: \mathrm{Yb}, \mathrm{PbTe}$. The normalization takes into account the time acquisition and the photoemission intensity marked by intensity of the anion core lines. The difference between the $\mathrm{PbTe}$ and $\mathrm{PbTe}: \mathrm{Yb}$ VB spectra is most pronounced near the Fermi level. The shape of the valence electrons energy distribution with two prominent peaks is characteristic for divalent $\mathrm{Yb}$ and corresponds to the $4 f^{14} \rightarrow f^{13}$ photoionization process $[5,6]$. These peaks can be assigned to the two states ${ }^{2} F_{7 / 2}$ and ${ }^{2} F_{5 / 2}$. The energy positions of maxima are $0.95 \mathrm{eV}$ and $2.3 \mathrm{eV}$, respectively, the spin-orbit splitting of the $4 f$ doublet is equal to $1.35 \mathrm{eV}$. This is typical value for the $4 f$ electrons distribution in the divalent $\mathrm{Yb}[5,6]$. Due to a large photoionization cross section for the $\mathrm{Yb} 4 f$ states, their influence on the valence band spectrum is distinctly seen, despite of the small $\mathrm{Yb}$ content.

Figure 2 presents the VB photoemission spectra of $\mathrm{PbS}: \mathrm{Yb}, \mathrm{PbS}$ and their difference. In comparison to $\mathrm{PbTe}: \mathrm{Yb}$ the doublet characteristic for divalent $\mathrm{Yb}$ corresponding to $4 f^{14}$ initial states is shifted towards the Fermi level and some additional structure appears at the energy $7-9 \mathrm{eV}$. This structure is caused by the existence of a trivalent $\mathrm{Yb}$ and corresponds to the $4 f^{13}$ initial states.

Similar difference between $\mathrm{PbS}: \mathrm{Yb}$ and $\mathrm{PbTe}: \mathrm{Yb}$, indicating a divalent character of $\mathrm{Yb}$ in $\mathrm{PbTe}$ and mixing valence in $\mathrm{PbS}$ is observed in $\mathrm{Yb} 4 d$ spectra. $\mathrm{Yb} 4 d$ spectrum of $\mathrm{PbTe}: \mathrm{Yb}$ consists of 2 peaks with energies 181 and $191 \mathrm{eV}$ 


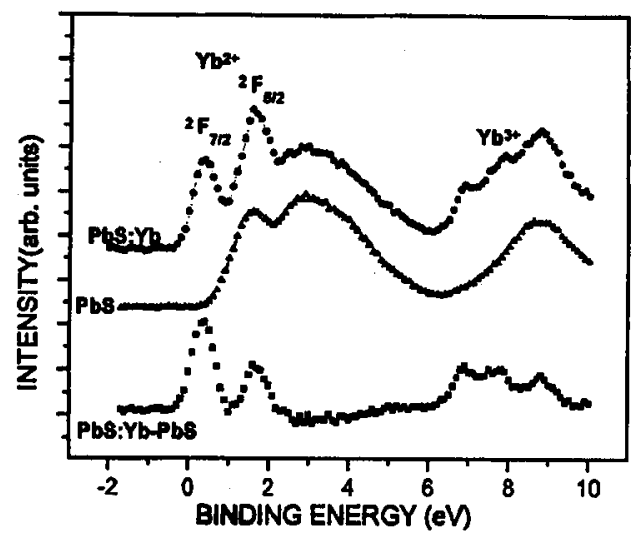

Fig. 2. XPS valence band spectra for $\mathrm{PbS}$ and $\mathrm{PbS}: \mathrm{Yb}$. The difference between the both spectra after normalization (PbS: $\mathrm{Yb}-\mathrm{PbS}$ ) is marked by the square points.

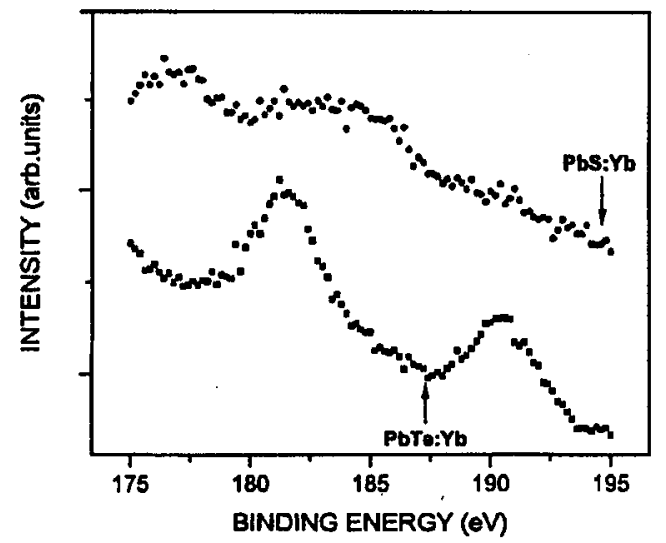

Fig. 3. Photoemission spectra of $\mathrm{Yb} 4 d$ core level in $\mathrm{PbTe}: \mathrm{Yb}$ and $\mathrm{PbS}: \mathrm{Yb}$.

corresponding to the $4 d^{5 / 2}$ and $4 d^{3 / 2}$ states, respectively (see Fig. 3). A similar position of the $4 d$ maxima -181.4 and $190.3 \mathrm{eV}$ of divalent $\mathrm{Yb}$ was observed in $\mathrm{Yb}_{4} \mathrm{Bi}_{3}[7]$.

For $\mathrm{PbS}: \mathrm{Yb}$ an additional broad structure appears with a binding energy of 180-187 eV. This spectrum is the superposition of two structures from divalent and trivalent $\mathrm{Yb}$. These positions of $4 d$ maxima of trivalent $\mathrm{Yb}$ observed in $\mathrm{YbAs}$ are approximately $183 \mathrm{eV}$ and $197 \mathrm{eV}$ [8].

In conclusion we found that the behaviour of $\mathrm{Yb}$ is different in $\mathrm{PbTe}$ and $\mathrm{PbS}$. This conclusion is supported by the analysis of the transport properties of both crystals. $\mathrm{PbTe}: \mathrm{Yb}$ is $p$-type semiconductor, while $\mathrm{Yb}$ doping causes $n$-type conductivity of $\mathrm{PbS}: \mathrm{Yb}$. 


\section{References}

[1] N.B. Brandt, V.V. Moshchalkov, Adv. Phys. 33, 374 (1984).

[2] C.M. Varma, Rev. Mod. Phys. 48, 219 (1976).

[3] G.R. Stewart, Rev. Mod. Phys. 56, 755 (1984).

[4] R.B. Shalvoy, G.B. Fisher, P.J. Stiles, Phys. Rev. B 15, 2021 (1977).

[5] K.H.J. Buschow, M. Campagna, G.K. Wertheim, Solid State Commun. 24, 253 (1977).

[6] R. Nyholm, Ib. Chorkendorf, J. Schmidt-May, Surf. Sci. 143, 177 (1984).

[7] S. Suga, S. Ogawa, H. Namatame, M. Taniguchi, A. Kakizaki, T. Ishii, A. Fujimori, S.-J. Oh, H. Kato, T. Miyahara, A. Ochiai, T. Suzuki, T. Kasuya, J. Phys. Soc. Jpn. 58, 4534 (1989).

[8] Jin-Seok Chung, En-Jin Cho, S.-J. Oh, Phys. Rev B 41, 5524 (1990). 